\title{
Neighbourhood and consumer food environment is associated with dietary intake among Supplemental Nutrition Assistance Program (SNAP) participants in Fayette County, Kentucky
}

\author{
Alison Gustafson ${ }^{1, *}$, Sarah Lewis ${ }^{1}$, Sarah Perkins ${ }^{1}$, Corey Wilson ${ }^{1}$, Elizabeth Buckner ${ }^{2}$ \\ and Ann Vail ${ }^{3}$ \\ 'Department of Dietetics and Human Nutrition, School of Human and Environmental Health, University of \\ Kentucky, Lexington, KY 40506, USA: ${ }^{2}$ School of Human Environmental Science, University of Kentucky, \\ Lexington, KY, USA: ${ }^{3}$ School of Human Environmental Science \& Family and Consumer Sciences Extension, \\ University of Kentucky, Lexington, KY, USA
}

Submitted 6 September 2012: Final revision received 1 January 2013: Accepted 22 January 2013: First published online 6 March 2013

\begin{abstract}
Objective: The aim of the study was to determine the association between dietary outcomes and the neighbourhood food environment (street network distance from home to stores) and consumer food environment (Nutrition Environment Measurement Survey-Stores (NEMS-S) audit).

Design: The neighbourhood food environment was captured by creating $0 \cdot 5$-mile and 1-mile network distance (street distance) around each participant's home and the nearest food venue (convenience store, grocery store, supermarket, farmers' market and produce stand). The consumer food environment was captured by conducting NEMS-S in all grocery stores/supermarkets within 0.5 and 1 mile of participants' homes.

Setting: Fayette County, KY, USA.

Subjects: Supplemental Nutrition Assessment Program (SNAP) participants, $n 147$. Results: SNAP participants who lived within 0.5 mile of at least one farmers' market/produce stand had higher odds of consuming one serving or more of vegetables $(\mathrm{OR}=6.92 ; 95 \% \mathrm{CI} 4.09,11.69)$, five servings or more of grains $(\mathrm{OR}=1 \cdot 76 ; 95 \% \mathrm{CI} 1 \cdot 01,3 \cdot 05)$ and one serving or more of milk (OR $=3 \cdot 79$; $95 \%$ CI $2 \cdot 14,6 \cdot 71)$ on a daily basis. SNAP participants who lived within 0.5 mile of stores receiving a high score on the NEMS-S audit reported higher odds of consuming at least one serving of vegetables daily $(\mathrm{OR}=3 \cdot 07 ; 95 \% \mathrm{CI}$ $1 \cdot 78,5 \cdot 31)$.

Conclusions: Taken together, both the neighbourhood food environment and the consumer food environment are associated with a healthy dietary intake among SNAP participants.
\end{abstract}

Keywords

NEMS-S

Food environment

SNAP

Diet
It has been well documented that low-income individuals have higher rates of obesity ${ }^{(1)}$ and lower intake of fruits and vegetables ${ }^{(2)}$. One particular group of low-income individuals who receive nutrition education programming and federal assistance to improve purchasing power is those participating in the Supplemental Nutrition Assistance Program (SNAP, formerly the Food Stamp Program) ${ }^{(3)}$. The aim of SNAP is to promote the general welfare and to safeguard the health and well-being of the US population by raising the levels of nutrition among low-income households $^{(4)}$. Between 2008 and 2011 there has been a steady increase in average household participation; from 12 million households per month in 2008 increasing to 22 million households per month in $2011^{(5)}$. However, accompanying the expansion of such programmes has been a rise in obesity rates that has led researchers to postulate that food insecurity and obesity may be intertwined. The connection between socio-economic status and health outcomes has led to the term 'food insecurity-obesity paradox ${ }^{,(6,7)}$. It has been suggested that perhaps the food vouchers SNAP participants receive might encourage them to consume more food compared with cash assistance ${ }^{(8)}$. Several studies have found significant associations between SNAP participation and higher $\mathrm{BMI}^{(9-12)}$ while others have found the opposite ${ }^{(13)}$.

Given these equivocal findings, it is unknown whether SNAP is achieving the original goal of improving nutritional outcomes of participants ${ }^{(6,14,15)}$. However, layered within this paradigm is the complex intersection between SNAP participants and the food resources available to low-income consumers. To date, there are limited studies addressing the role of the food environment as a determinant in diet and 
weight among SNAP participants ${ }^{(16,17)}$. However, it has been well documented that neighbourhoods with a higher proportion of minority or low-income residents have fewer healthy food retailers ${ }^{(18-20)}$ and less availability of healthy foods within stores ${ }^{(21,22)}$. Specifically, recent findings indicate that among SNAP participants BMI is significantly lower if they have access to more supermarkets/grocery stores ${ }^{(16)}$. Moreover, recent reviews have indicated that spatial availability of certain food venues is associated with dietary outcomes among low-income populations ${ }^{(23-25)}$.

Yet only recently has research begun to examine how simultaneous measurement of the neighbourhood food environment (food stores close to where individuals live) and the consumer food store environment (availability, price, promotion, quality, placement of food within stores where individuals shop) ${ }^{(23,26)}$ relates to the continuum of socio-economic status and dietary habits. Of the studies addressing the relationship between food store access and diet, results have shown among low-income consumers that access to fast-food restaurants is associated with intake ${ }^{(27-29)}$. However, other studies have reported that access to healthy food venues, such as supermarkets, is not associated with diet or weight ${ }^{(30,31)}$. These results suggest that perhaps the effect of being close to less healthy options may promote intake of high-energy foods, rather than access to healthy stores promoting intake of lower-energy foods.

Policy-level approaches have begun to address improving access to different food venues as a way to improve intake among those participating in $\mathrm{SNAP}^{(32)}$. One approach has been for farmers' markets to install the equipment necessary to accept electronic benefit transfer (EBT) cards. By having the equipment and financial agreement with the federal government to accept EBT cards at each vendor within the farmers' market, the approach posits that more SNAP participants will utilize and purchase fresh fruits and vegetables at these markets since their EBT cards are readily accepted. Results from this policy approach have shown an increase in the number of SNAP participants purchasing fruits and vegetables at farmers' markets ${ }^{(33)}$. However, little is still known about how the neighbourhood food environment is associated with dietary intake and diet quality among those receiving SNAP benefits ${ }^{(34)}$.

The consumer food environment addresses the food environment within stores where consumers shop ${ }^{(35)}$. At the same time that the neighbourhood food environment is important to improve access to food venues selling food, the consumer food environment is equally relevant $^{(35)}$. To date studies have found that the availability of healthy food within stores may or may not be associated with dietary intake of fruits and vegetables ${ }^{(36,37)}$. The conflicting results may be more a reflection of the methods used, the sample populations and also the reality that supermarkets sell more produce but they also sell more unhealthy items at the same time ${ }^{(38)}$. When food shopping, individuals face the decision to purchase a healthy food item at the same time they are faced with the decision to buy an unhealthy food item. Although consumers receiving SNAP benefits may purchase fruits and vegetables, they may also purchase less healthy items which leads to an overall overconsumption of energy. Thus measuring the overall availability, price and quality of food within stores is needed to understand the association between the consumer food environment and diet among low-income consumers.

Taken together, the neighbourhood-level availability of food venues as well as the consumer food environment may have distinct or complementary influences on dietary habits among lower-income adults. To assess the neighbourhood food store environment as well the effect of the consumer food store environment on dietary intake, the aims of the present study were to determine the associations between: (i) the neighbourhood food environment (number of stores within network distances of 0.5 mile and 1 mile from home) and dietary outcomes; and (ii) the consumer food environment (Nutrition Environment Measurement Survey-Store (NEMS-S) audit) in stores within 0.5 mile and 1 mile from home and dietary outcomes. The hypothesis was that those who live in an area with more farmers' markets, produce stands and supermarkets would report higher consumption of healthy food items compared with those who live in an area with fewer farmers' markets, produce stands and supermarkets. The second hypothesis was that those who live in an area where stores have higher availability of healthy foods would report higher consumption of healthy food items compared with those who live in an area where stores have a low availability of healthy foods.

\section{Methods}

\section{Study region and design}

The spatial area under analysis was Fayette County, KY, USA (population $=295803$ ). Fayette County covers an area of 283 square miles with approximately 1000 people/square mile, with a small urban centre as the county seat. The racial and ethnic composition of Fayette County is $76 \%$ white, $15 \%$ black, $7 \%$ Hispanic or Latino and 2\% Asian based on the US Census Bureau 2010 ${ }^{(39)}$. The study was reviewed and determined as exempt by the University of Kentucky Internal Review Board, because it was a secondary data analysis with data that are publicly available to the University.

The study design was a cross-sectional survey assessing dietary intake and food shopping behaviours. The study took place between January 2010 and January 2011.

\section{Study sample}

Limited-resource families were identified to participate in the SNAP-Ed programme through county Cooperative Extension offices. To be eligible to participate in SNAP, an 
individual's per capita family income (adjusted for family size) must be less than or equal to $130 \%$ of the federal poverty guideline.

To be eligible for the present analysis, individuals needed to participate in SNAP-Ed in Kentucky during the years 2010-2011. Individuals were excluded from analysis if baseline data were missing ( $n$ 2).

\section{Recruitment}

Limited-resource families were recruited through local county agencies, health departments, SNAP benefit offices and commodity distribution programmes. Adults aged 18 years and over with complete information on all independent, dependent and covariates (complete cases) were included for analysis ( $n$ 147).

\section{Neighbourbood food environment}

Food venues were identified via a commercial database. Food venue addresses for Fayette County were purchased from InfoUSA database in July 2011. Addresses were categorized based on North American Industry Classification System (NAICS) codes. The categories of interest based on consumer shopping behaviours were as follows: supermarkets/grocery stores (Group 445100), convenience stores (446110) and gas stations with food marts (447110). Farmers' markets and produce stands were identified through the health department's listing of such vendors. Farmers' markets were verified through the Kentucky Department of Agriculture.

The neighbourhood food environment was captured by creating $0 \cdot 5$-mile and 1-mile network distances (street distance) around each participant's home and counting the number of convenience stores and gas stations, grocery stores and supermarkets, and farmers' markets and produce stands within these network distances. Network distance is a set of interconnected lines whose attributes share a common theme. Network distance relates to the connectivity of travel routes from an 'origin to a specific destination, ${ }^{(40)}$. Using ArcGIS an individual's home address was mapped within Lexington, KY. Next, using the network analyst function within ArcGIS, a street connection rather than a straight line was used to link the individual's home address with the nearest food venue for each sub-type of store (convenience, grocery, supermarket, farmers' market, produce stand). These various lines were then used to create 0.5 - and 1.0-mile areas around the individual's home. Lastly, each store type was counted within these areas to derive the neighbourhood food store environment. Based on the distribution of the data, convenience stores and gas stations, grocery stores and supermarkets, and farmers' markets and produce stands were all categorized as 0 if no store was within 0.5 mile of the participant's home and as 1 if one or more of the store type was within 0.5 mile. For the network distance of 1 mile the following categories were used: convenience stores and gas stations $(\leq 4 v .>4)$, grocery stores and supermarkets ( $\leq 8 v .>8)$, and farmers' markets and produce stands $(0 v, \geq 1)$. These categories were created based on the mean value for each food venue type. For the network distance of 0.5 mile the reference was zero since many individuals did not have stores within half a mile from their home.

\section{Consumer food environment}

The consumer food store environment was captured by conducting an audit within the stores where SNAP participants did their primary food shopping. Since it was not known where SNAP participants shopped, the consumer food environment was captured by conducting the NEMS-S in all the major supermarkets within 1.0-mile network distance around participants' homes. A total of nine retail outlets (large grocery/supermarket stores based on NAICS code 445100 ) were within $1 \cdot 0$-mile network distance around all SNAP participants' home. These large grocery stores/supermarkets were also selected since close to $90 \%$ of SNAP participants redeem their benefits at large grocery stores or supermarkets ${ }^{(41,42)}$. A total of four grocery stores were within 0.5 mile from the participants' homes.

Food store audits using the Nutrition Environment Measurement Survey-Stores (NEMS-S) ${ }^{(43)}$ with slight modifications (NEMS-S Rudd) ${ }^{(44)}$ were used to assess the consumer food environment. The NEMS-S Rudd is a modified version of the original NEMS-S and includes tuna, canned and frozen vegetables, rice and pasta. The modifications consisted of adding two store-prepared meal options (rotisserie and fried chicken meals, mixed green salad and mayonnaise-based vegetable salad) and three snack food items (low-fat and regular potato chips, regular and reduced-fat cookies, ice cream and reducedfat ice cream) based on trends in consumption ${ }^{(45)}$ and previous research ${ }^{(46,47)}$. The store audits were conducted on Monday to Friday between 09.00 and 17.00 hours by graduate students trained in the NEMS-S protocol. NEMS-S online training certification ${ }^{(43)}$ was obtained by the principal investigator and subsequent trainings were given to the graduate students conducting the assessments. A total of fifteen food categories and fifty-five unique food items were assessed. Availability and price were collected for all food items, while quality was collected on all produce items. Availability was recorded as well as price per unit or ounce on the NEMS-S protocol audit sheet and quality was measured as per NEMS-S protocol $^{(43)}$. The NEMS-S is scored to provide a composite score for the different subscales (healthy availability, pricing and quality) ${ }^{(43)}$. The total NEMS-S score ranged from 0 to 80 , availability was scored based on the protocol with a possible range in scores of $0-55$, price was scored based on NEMS-S protocol with a possible range in scores of $0-15$, and quality with a range of $0-10$. Higher NEMS-S scores indicated stores with better availability, price and quality of healthy foods. Two categorical variables were created for NEMS-S based on the distribution of the data. 
The mean score was used to create high and low categories for stores within the $0 \cdot 5$-mile network distance $(<21 v$. $\geq 21)$ and 1-mile network distance $(<50 v$. $\geq 50)$.

\section{Diet quality - Healtby Eating Index score}

A $24 \mathrm{~h}$ dietary recall was obtained from every participant by a Cooperative Extension agent trained in data collection. The type and amount of foods consumed were recalled using aids such as abstract food models, special charts, measuring cups and rulers to help in quantifying the amounts consumed. Special probes were used to help the recall of commonly forgotten items such as condiments, accompaniments and fast food. Based on these data, dietary habits as well as the dietary intakes of protein, carbohydrate, fat and total energy were quantified using the nutrition software NutWin ${ }^{(48)}$.

Dietary intake data, as assessed by the $24 \mathrm{~h}$ recall, were used to calculate Healthy Eating Index (HEI) scores. The HEI comprises ten components (saturated fat, total fat, cholesterol, sodium, grain, fruit, dairy, meat and vegetable intakes, plus a measure of dietary variety), each contributing 10 points to the maximum possible score of $100^{(49)}$. Food serving amounts were computed from food consumption data using factors derived from the servingsize assumptions given in the US Department of Agriculture Food Guide Pyramid. Ten points were awarded if saturated fat $\leq 10 \%$ of energy, total fat $\leq 30 \%$ of energy, cholesterol $\leq 300 \mathrm{mg}$ and sodium $\leq 2 \cdot 4 \mathrm{mg}$. A zero score reflected $\geq 15 \%$ of energy for saturated fat, $\geq 45 \%$ of energy for total fat, cholesterol $\geq 450 \mathrm{mg}$ and sodium $\geq 4.8 \mathrm{mg}$; between these two cut-off points, scores were scaled proportionately. A similar process was used for food groups (grains, fruits, vegetables, dairy and meat): consumption of the recommended number of servings for the individual's age and sex resulted in a score of 10, with a score of 0 if no servings were consumed. A variety component was also used with a scoring method of $\geq 8$ food items $=10$ points and $<8=0$ points. The maximum overall score for the ten components combined was 100 .

For each food group listed above a dichotomous variable was created based on the distribution of the data. Sensitivity tests were conducted for various cut-off points and the following categories were most appropriate based on the distribution of the data. Fruit, vegetable and milk categories were created for $<1$ serving and $\geq 1$ serving consumed per day. Meat and grains were categorized as $<5$ five servings and $\geq 5$ servings consumed per day.

\section{Statistical analysis}

Descriptive statistics were calculated as means and standard deviations, or proportions. To test for differences between group means, the $t$ test was used. To test the associations between each dietary outcome and neighbourhood food environment variables (0.5-mile and 1-mile network distance for each store type) and the consumer food environment (NEMS-S scores in stores), multivariate logistic regression was used. Multilevel analyses were tested using a hierarchical linear model; however, the interclass correlation coefficient was 0.02 and thus a hierarchical linear model was not appropriate. Additionally, there were a few clusters due to the small sample size; therefore to achieve robust standard errors a cluster command on zip code was used for all models. The likelihood ratio test was used to test for model fit. Type I error rate was set at $\alpha=0 \cdot 05$. All models were adjusted for age, race, gender, number of children in the household and population density.

To test the associations between HEI scores, neighbourhood food environment and consumer food environment, multivariate linear regression was used. The Shapiro-Wilk test for normality was used for model fit. Type I error rate was set at $\alpha=0 \cdot 05$. All models were adjusted for age, race, gender, number of children in the household and population density. All analyses were conducted with the Stata statistical software package version $11 \cdot 0$.

\section{Results}

The study sample ( $n$ 147) was $49 \%$ female, $65 \%$ had no children in the household and their average age was 37 years (Table 1 ). The sample on average consumed less than one serving of fruit and a little over one serving of vegetables on a daily basis. There were no statistically significant differences between the number of convenience stores/gas stations, grocery stores/supermarkets and farmers' markets/produce stands within 0.5 mile of the participants' homes $(P=0 \cdot 058)$. However, there were significant differences between the number of stores according to type within 1 mile of the participants' homes $(P=0 \cdot 009)$. There were more grocery/supermarkets and convenience stores/gas stations within 1 mile of home relative to farmers' markets/produce stands. Lastly, the stores within 0.5 mile of participants' homes had significantly lower scores for availability of healthy foods compared with the stores within 1 mile of the participants' homes $(P=0 \cdot 02)$.

\section{Half-mile network distance}

SNAP participants who lived in a neighbourhood within a 0.5-mile network distance to at least one convenience store or gas station had lower odds of consuming five servings or more of meat daily compared with those having no convenience store or gas stations. SNAP participants who lived within a $0 \cdot 5$-mile network distance to at least one farmers' market or produce stand had higher odds of consuming one serving or more of vegetables $(\mathrm{OR}=6.92 ; 95 \% \mathrm{CI} 4.09,11 \cdot 69)$, five servings or more of grains $(\mathrm{OR}=1 \cdot 76 ; 95 \% \mathrm{CI} 1 \cdot 01,3 \cdot 05)$, one serving or more of milk $(\mathrm{OR}=3 \cdot 79 ; 95 \% \mathrm{CI} 2 \cdot 14,6 \cdot 71)$ and five servings or more of meat $(\mathrm{OR}=3 \cdot 34 ; 95 \% \mathrm{CI} 2 \cdot 06,5 \cdot 43)$ on a daily basis compared with those who did not live within 0.5 mile of any farmers' market or produce stand. 
Table 1 Characteristics of the SNAP participants ( $n$ 147), neighbourhood food environment and consumer food environment, Fayette County, KY, USA, 2010-2011

\begin{tabular}{|c|c|c|c|c|}
\hline & $\%$ & Mean & SD & $P$ value \\
\hline \multicolumn{5}{|l|}{ Gender } \\
\hline Female & 49 & & & \\
\hline Male & 51 & & & \\
\hline \multicolumn{5}{|l|}{ Race } \\
\hline White & 67 & & & \\
\hline African American & 25 & & & \\
\hline Other & 8 & & & \\
\hline \multicolumn{5}{|l|}{ Number of children in household } \\
\hline 0 & 65 & & & \\
\hline 1 & 22 & & & \\
\hline $2-3$ & 10 & & & \\
\hline$\geq 4$ & 3 & & & \\
\hline Age (years) & & 37 & $13 \cdot 13$ & \\
\hline \multicolumn{5}{|l|}{ Dietary intake (servings/d) } \\
\hline Fruits & & 0.68 & 0.07 & \\
\hline Vegetables & & $1 \cdot 63$ & $0 \cdot 10$ & \\
\hline Grains & & $5 \cdot 49$ & 0.33 & \\
\hline Milks & & $1 \cdot 38$ & $0 \cdot 11$ & \\
\hline Meat & & $4 \cdot 97$ & 0.33 & \\
\hline \multicolumn{5}{|l|}{ HEl score } \\
\hline Total & & $59 \cdot 03$ & $1 \cdot 16$ & \\
\hline Variety & & $6 \cdot 79$ & 0.30 & \\
\hline \multicolumn{5}{|c|}{ Neighbourhood food environment, 0.5 mile } \\
\hline Convenience stores/gas stations & $42^{*}$ & 0.54 & 0.06 & 0.058 \\
\hline Farmers' markets/produce stands & $53^{\star}$ & 0.54 & 0.06 & \\
\hline Grocery stores/supermarkets & $76^{\star}$ & $1 \cdot 61$ & $0 \cdot 13$ & \\
\hline \multicolumn{5}{|c|}{ Neighbourhood food environment, 1 milet } \\
\hline Convenience stores/gas stations & & $5 \cdot 31$ & 0.22 & 0.009 \\
\hline Farmers' markets/produce stands & & $1 \cdot 12$ & $0 \cdot 10$ & \\
\hline Grocery stores/supermarkets & & $7 \cdot 37$ & 0.29 & \\
\hline \multicolumn{5}{|l|}{ Consumer food environment } \\
\hline NEMS-S score, 0.5 mile & & $21 \cdot 27$ & $1 \cdot 70$ & 0.02 \\
\hline NEMS-S score, 1 mile & & $50 \cdot 92$ & $1 \cdot 00$ & \\
\hline
\end{tabular}

SNAP, Supplemental Nutrition Assessment Program; HEI, Healthy Eating Index; NEMS-S, Nutrition Environment Measurement Survey-Stores.

${ }^{*}$ Percentage of participants with at least one of this store type within 0.5 mile of home.

tAll SNAP participants have at least one store type within 1 mile from home.

Also, living within 0.5 mile of at least one grocery store or supermarket was associated with higher odds of consuming at least one serving of vegetables daily, one serving or more of milk daily and five servings or more of grains and meat daily compared with those who had no grocery stores or supermarkets within 0.5 mile of home (Table 2). The overall diet score based on the HEI was not associated with proximity at 0.5 mile for convenience stores/gas stations or grocery stores/supermarkets. However, those who lived within 0.5 mile of a farmers' market or produce stand scored about two points higher for variety in their diet compared with those who did not have any farmers' markets or produce stands in their neighbourhood $(\beta=2 \cdot 17 ; 95 \%$ CI $0 \cdot 12,4 \cdot 23)$.

\section{One-mile network distance}

The results were similar for SNAP participants who lived in a neighbourhood within a 1-mile network distance to farmers' markets/produce stands or grocery stores/ supermarkets. However, the results were different for those living within 1 mile of a convenience store or gas station. Living within 1 mile of more than four convenience stores/gas stations was associated with higher odds of consuming at least one serving or more of fruit, vegetables and milk and five servings or more of meat on a daily basis (Table 2).

SNAP participants who lived within 1 mile of more than four convenience stores/gas stations scored a little over three points higher on the HEI score compared with those having fewer convenience stores and gas stations within 1 mile of home $(\beta=3 \cdot 69 ; 95 \%$ CI $0 \cdot 32,7 \cdot 07)$. With regard to HEI sub-score of variety, results indicated that those who lived within 1 mile of at least four convenience stores/gas stations scored higher for variety in their diet compared with those with fewer convenience stores/gas stations $(\beta=1 \cdot 79 ; 95 \%$ CI $0 \cdot 75,2 \cdot 83)$ within 1 mile from their home. Those who lived within 1 mile to a farmers' market or produce stand scored one point higher for variety in their diet compared with those who did not $(\beta=1 \cdot 36$; $95 \%$ CI $0 \cdot 18,2 \cdot 54$ ). Lastly, those who lived within 1 mile of at least eight grocery stores/supermarkets scored over two points higher on variety of HEI compared with those who had fewer than eight grocery stores/supermarkets within 1 mile of home $(\beta=2 \cdot 17 ; 95 \%$ CI $0 \cdot 12,4 \cdot 23)$. 


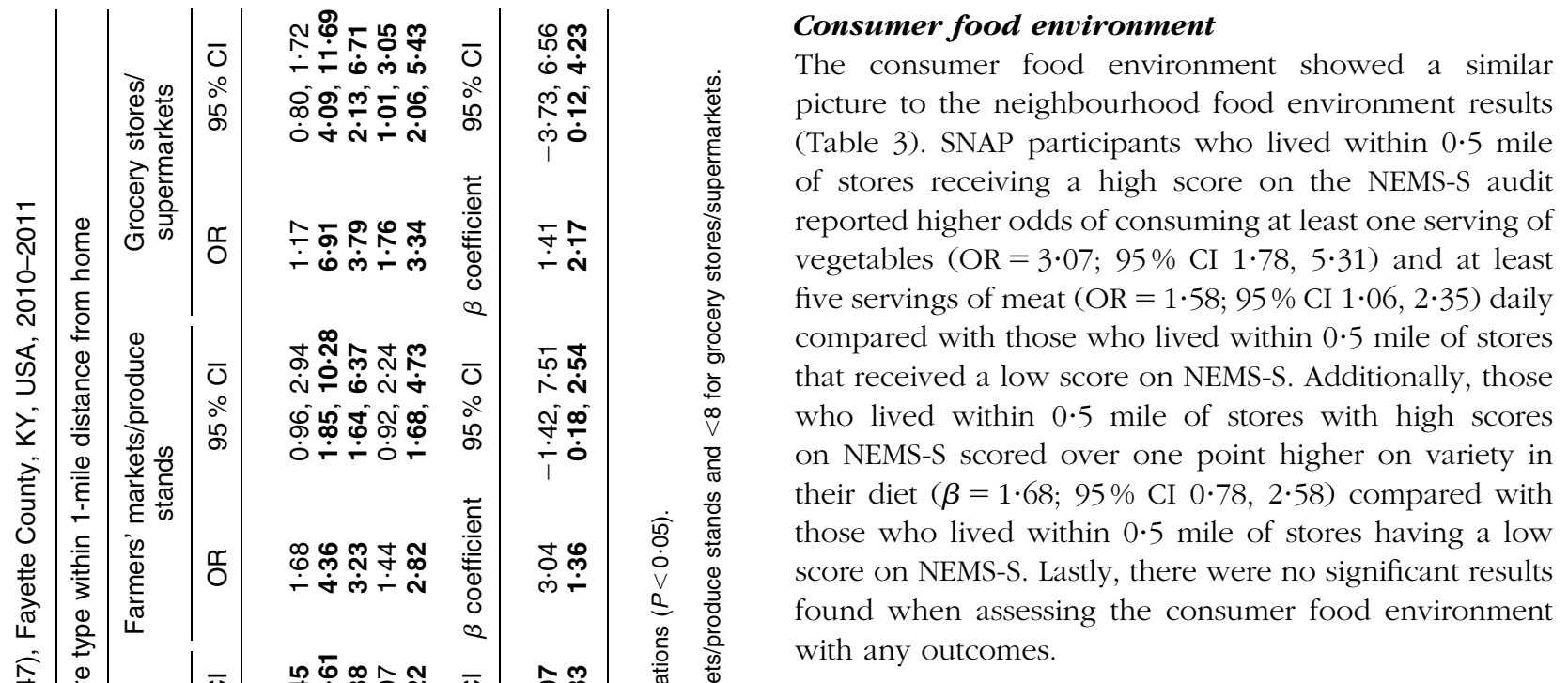

Consumer food environment

The consumer food environment showed a similar picture to the neighbourhood food environment results (Table 3). SNAP participants who lived within 0.5 mile of stores receiving a high score on the NEMS-S audit reported higher odds of consuming at least one serving of vegetables (OR $=3 \cdot 07 ; 95 \% \mathrm{CI} 1 \cdot 78,5 \cdot 31)$ and at least who lived within 0.5 mile of stores with high scores on NEMS-S scored over one point higher on variety in their diet $(\beta=1 \cdot 68 ; 95 \%$ CI $0 \cdot 78,2 \cdot 58)$ compared with those who lived within 0.5 mile of stores having a low score on NEMS-S. Lastly, there were no significant results with any outcomes.

\section{Discussion}

The study results indicated that, overall, both the neighbourhood food environment and the consumer food environment are associated with dietary intake among SNAP participants. Those who lived within 0.5 mile of farmers' markets, produce stands, grocery stores and supermarkets consumed more healthy food items than those who lived in neighbourhoods with fewer of these types of venues and stores with lower scores on the consumer food environment.

Previous studies have indicated mixed results with respect to associations between proximity to stores selling healthy food items and dietary intake ${ }^{(21,22,30,50)}$. Yet, in most studies farmers' markets and produce stands have not been included. The addition of this type of food venue allows for understanding how proximity to various store types, rather than one single source, may influence intake $^{(51)}$. In all models testing proximity to farmers' markets and produce stands with dietary intake, there were significant findings between proximity and all dietary outcomes and variety in the dietary score. Among SNAP participants the ability to use EBT cards at farmers' markets may allow for use of these venues and aid in providing a mix of foods that would otherwise not be purchased $^{(33,52)}$.

Although a recent review indicated that there is still much to be learned about the introduction of farmers' markets into communities and the effect on dietary outcomes, early studies are promising ${ }^{(34)}$. One study reported that low-income participants receiving the Special Supplemental Nutrition Program for Women, Infants, and Children who utilized the farmer's market consumed more vegetables ${ }^{(53)}$. Recently an intervention introducing farm stands in low-income communities reported increased consumption of fruits and vegetables among low-income residents within the neighbourhood ${ }^{(54)}$. Yet, there still remain few 
Table 3 Consumer food environment and the association with dietary intake and diet quality of SNAP participants (n 147), Fayette County, KY, USA, 2010-2011

\begin{tabular}{|c|c|c|c|c|}
\hline & \multicolumn{2}{|c|}{ NEMS-S score, 0.5 mile (four stores) } & \multicolumn{2}{|c|}{ NEMS-S score, 1 mile (nine stores) } \\
\hline & OR & $95 \% \mathrm{Cl}$ & OR & $95 \% \mathrm{Cl}$ \\
\hline \multicolumn{5}{|c|}{ Dietary intake (servings/d) } \\
\hline Fruits & $1 \cdot 15$ & $0 \cdot 75,1 \cdot 80$ & $0 \cdot 69$ & $0.33,1.47$ \\
\hline Vegetables & 3.07 & $1 \cdot 78,5 \cdot 31$ & $2 \cdot 04$ & $0.54,7 \cdot 71$ \\
\hline Grains & 0.98 & $0 \cdot 68,1 \cdot 42$ & $1 \cdot 15$ & $0 \cdot 58,2 \cdot 30$ \\
\hline Milk & $1 \cdot 62$ & $0 \cdot 81,3 \cdot 23$ & 1.02 & $0.59,1 \cdot 77$ \\
\hline \multirow[t]{2}{*}{ Meat } & $1 \cdot 58$ & $1 \cdot 06,2 \cdot 35$ & $1 \cdot 49$ & $0 \cdot 80,2 \cdot 77$ \\
\hline & $\beta$ coefficient & $95 \% \mathrm{Cl}$ & $\beta$ coefficient & $95 \% \mathrm{Cl}$ \\
\hline \multicolumn{5}{|l|}{ HEI score } \\
\hline Total & $0 \cdot 18$ & $-4 \cdot 36,4 \cdot 72$ & $-1 \cdot 59$ & $-8 \cdot 45,5 \cdot 27$ \\
\hline Variety & 1.68 & $0.78,2.58$ & 0.57 & $-0.64,1 \cdot 79$ \\
\hline
\end{tabular}

SNAP, Supplemental Nutrition Assessment Program; NEMS-S, Nutrition Environment Measurement Survey-Stores; HEI, Healthy Eating Index.

All models are adjusted for race, age, number of children in the household and population density. Bold font indicates statistically significant associations $(P<0.05)$.

Reference for fruits, vegetables and milk is $<1$ serving/d; reference for grains and meat is $<5$ servings/d.

Reference for NEMS-S score 0.5 mile is total score $<21$; reference for NEMS-S score 1 mile is total score $<50$.

well-designed studies assessing interventions and policies aimed at improving access and utilization of farmers' markets with dietary intake among low-income consumers ${ }^{(34)}$. Thus caution is warranted in interpreting findings from the present study, since it was a cross-sectional survey and causal inference cannot be established. Longitudinal studies are needed to establish the effect of non-traditional food venues as an approach to improving intake.

A secondary hypothesis was that those living close to convenience stores would consume less healthy items. The study findings did not find any association, other than for meat, between store proximity to convenience stores/gas stations and less consumption of healthy food. However, when the analyses assessed proximity of convenience stores/gas stations within 1 mile of participants' homes there was higher consumption of fruits, vegetables, milk and meat. This finding suggests that proximity to convenience stores/gas stations may not be associated with intake, but having fewer of these stores within 0.5 mile from residents' homes might force individuals to choose a different food venue with healthier food options. Other studies have found that proximity to convenience stores is associated with BMI among adolescents ${ }^{(55,56)}$, while others have found that proximity is not associated with $\mathrm{BMI}^{(57,58)}$. Specifically among SNAP participants, who may be more sensitive to proximity relative to other populations, a recent simulation study suggested that if these individuals were faced with more supermarkets and grocery stores they would have a lower BMI ${ }^{(16)}$. The authors suggested that policies aimed at improving access to supermarkets among SNAP participants may help improve dietary intake and thus BMI. Findings from our study corroborate this simulation and policy suggestion since SNAP participants who lived close to grocery stores and supermarkets consumed more vegetables, milk and grains per day relative to those who did not live within 0.5 mile of a grocery store or supermarket.
The consumer food environment results highlight the role that both consumer and neighbourhood determinants play in diet. SNAP participants whose residence was within 0.5 mile of food stores that had higher scores on the NEMS-S audits (availability + price + quality) consumed more vegetables and meat, and had higher score on the HEI variety subscale, compared with participants who lived within 0.5 mile of stores with low scores on NEMS-S. The results are similar to previous studies conducted among urban populations ${ }^{(37)}$ indicating that living in a neighbourhood with stores having higher NEMS-S score was associated with a better dietary score. These findings suggest that living close to a variety of stores selling healthy food is relevant but equally important is the food within stores where individuals shop and can use their SNAP benefits. The implications of this finding highlight the need for policy approaches at both the neighbourhood level and store level to provide healthy affordable food for low-income consumers.

There are several limitations to our study which are worth noting. The first limitation is that the exact location where the SNAP participants shopped was not collected. However, since we covered all of the stores within 0.5 mile and 1 mile of their home we can estimate that they might choose to shop at one of these stores. Individuals most likely shop outside a $0 \cdot 5$-mile or 1-mile network distance for food and because the study did not capture daily living patterns, future studies will be needed to address daily living patterns as a way to understand how low-income adults' daily patterns influence food shopping behaviours and dietary outcomes. Second, dietary intake was self-reported which is subject to misclassification. However, all participants were low-income and had the same agents collecting dietary data. Third, food stores may have closed from the time the survey was conducted and the audits were done. However, verification with the county health department did not indicate any store closings. Lastly, the small sample may 
have been under-powered to detect associations and thus findings need to be repeated in future studies.

\section{Conclusion}

The present findings suggest that providing access and availability to different food venues, such as farmers' markets and produce stands, is associated with a healthy dietary intake among SNAP participants. Communitylevel approaches might consider increasing the number of food venues available, but also the location or proximity to low-income consumers, as a way to improve access to fresh and affordable food. However, additional research is still needed utilizing valid and reliable dietary assessment methods to evaluate the influence of farmers' markets and community gardens on nutrition-related outcomes $^{(34)}$. Additionally, interventions need to target the consumer food environment within stores by promoting healthful eating through affordability, availability, prominence and promotion of key healthful foods ${ }^{(59)}$. Policies which support development of food venues in low-income neighbourhoods as well as collaboration with store owners in promotion and placement of healthful foods are two possible mechanisms to improve overall dietary intake among low-income consumers ${ }^{(60)}$.

\section{Acknowledgements}

Sources of funding: This study was funded by the University of Kentucky Research Foundation and the National Institute of Minority Health and Disparities of the National Institutes of Health. Conflicts of interest: The authors report no conflict of interest. Authors' contributions: A.G. conducted the data analysis, interpreted of the data, and was responsible for writing, revising and the decision of manuscript publication. S.L. assisted with data collection. S.P. assisted with data collection. C.W. conducted all network analyses using ArcGIS mapping software. E.B. assisted with revision of the manuscript. A.V. assisted with the conceptual design of the study.

\section{References}

1. Mokdad AH, Ford ES, Bowman BA et al. (2003) Prevalence of obesity, diabetes, and obesity-related health risk factors, 2001. JAMA 289, 76-79.

2. Middaugh AL, Fisk PS, Brunt A et al. (2012) Few associations between income and fruit and vegetable consumption. J Nutr Educ Behav 44, 196-203.

3. Kaufman P, MacDonald J, Lutz S et al. (1997) Do the Poor Pay More for Food? Item Selection and Price Differences Affect Low-Income Household Food Costs. Washington, DC: Food and Rural Economics Division, Economic Research Service, US Department of Agriculture.

4. Debono NL, Ross NA \& Berrang-Ford L (2012) Does the Food Stamp Program cause obesity? A realist review and a call for place-based research. Health Place 18, 747-756.
5. US Department of Agriculture (2011) Program Data. Supplemental Nutrition Assistance Program. Annual State Level Data. http://www.fns.usda.gov/pd/snapmain.htm (accessed January 2013).

6. Dinour LM, Bergen D \& Yeh MC (2007) The food insecurity-obesity paradox: a review of the literature and the role food stamps may play. J Am Diet Assoc 107, 1952-1961.

7. Dietz WH, Franks AL \& Marks JS (1998) The obesity problem. $N$ Engl J Med 338, 1157.

8. Fox MK, Hamilton WL \& Lin B-H (2004) Effects of Food Assistance and Nutrition Programs on Health. Executive Summary of the Literature Review. Washington, DC: US Department of Agriculture, Economic Research Service.

9. Townsend MS, Peerson J, Love B et al. (2001) Food insecurity is positively related to overweight in women. J Nutr 131, 1738-1745.

10. Leung CW \& Villamor E (2011) Is participation in food and income assistance programmes associated with obesity in California adults? Results from a state-wide survey. Public Health Nutr 14, 645-652.

11. Jilcott SB, Liu H, Dubose KD et al. (2011) Food stamp participation is associated with fewer meals away from home, yet higher body mass index and waist circumference in a nationally representative sample. J Nutr Educ Behav 43, 110-115.

12. Webb AL, Schiff A, Currivan D et al. (2008) Food Stamp Program participation but not food insecurity is associated with higher adult BMI in Massachusetts residents living in low-income neighbourhoods. Public Health Nutr 11, 1248-1255.

13. Ver Ploeg M, Mancino L, Lin BH et al. (2007) The vanishing weight gap: trends in obesity among adult food stamp participants (US) (1976-2002). Econ Hum Biol 5, 20-36.

14. Rose D \& Richards R (2004) Food store access and household fruit and vegetable use among participants in the US Food Stamp Program. Public Health Nutr 7, 1081-1088.

15. Ford PB \& Dzewaltowski DA (2011) Neighborhood deprivation, supermarket availability, and BMI in lowincome women: a multilevel analysis. J Community Health 36, 785-796.

16. Han E, Powell LM \& Isgor Z (2012) Supplemental nutrition assistance program and body weight outcomes: the role of economic contextual factors. Soc Sci Med 74, 1874-1881.

17. Jilcott SB, Moore JB, Wall-Bassett ED et al. (2011) Association between travel times and food procurement practices among female supplemental nutrition assistance program participants in eastern North Carolina. J Nutr Educ Behav 43, 385-389.

18. Morland K \& Filomena S (2007) Disparities in the availability of fruits and vegetables between racially segregated urban neighbourhoods. Public Health Nutr 10, 1481-1489.

19. Bodor JN, Rice JC, Farley TA et al. (2010) The association between obesity and urban food environments. J Urban Health 87, 771-781.

20. Schulz AJ, Zenk SN, Israel BA et al. (2008) Do neighborhood economic characteristics, racial composition, and residential stability predict perceptions of stress associated with the physical and social environment? Findings from a multilevel analysis in Detroit. J Urban Health 85, 642-661.

21. Izumi BT, Zenk SN, Schulz AJ et al. (2011) Associations between neighborhood availability and individual consumption of dark-green and orange vegetables among ethnically diverse adults in Detroit. $J$ Am Diet Assoc 111, 274-279.

22. Zenk SN, Lachance LL, Schulz AJ et al. (2009) Neighborhood retail food environment and fruit and vegetable intake in a multiethnic urban population. Am J Health Promot 23, 255-264.

23. Gustafson A, Hankins S \& Jilcott S (2012) Measures of the consumer food store environment: a systematic review of the evidence 2000-2011. J Community Health 37, 897-911. 
24. McKinnon RA, Reedy J, Morrissette MA et al. (2009) Measures of the food environment: a compilation of the literature, 1990-2007. Am J Prev Med 36, 4 Suppl., S124-S133.

25. Caspi CE, Sorensen G, Subramanian SV et al. (2012) The local food environment and diet: a systematic review. Health Place 18, 1172-1187.

26. Thornton LE, Crawford DA \& Ball K (2010) Neighbourhood-socioeconomic variation in women's diet: the role of nutrition environments. Eur J Clin Nutr 64, 1423-1432.

27. Lucan SC, Barg FK \& Long JA (2010) Promoters and barriers to fruit, vegetable, and fast-food consumption among urban, low-income African Americans - a qualitative approach. Am J Public Health 100, 631-635.

28. Pearce J, Blakely T, Witten K et al. (2007) Neighborhood deprivation and access to fast-food retailing: a national study. Am J Prev Med 32, 375-382.

29. Fleischhacker SE, Evenson KR, Rodriguez DA et al. (2011) A systematic review of fast food access studies. Obes Rev 12, e460-e471.

30. Boone-Heinonen J, Gordon-Larsen P, Kiefe CI et al. (2011) Fast food restaurants and food stores: longitudinal associations with diet in young to middle-aged adults: the CARDIA Study. Arch Intern Med 171, 1162-1170.

31. Block JP, Christakis NA, O'Malley AJ et al. (2011) Proximity to food establishments and body mass index in the Framingham Heart Study offspring cohort over 30 years. Am J Epidemiol 174, 1108-1114.

32. Story M, Kaphingst KM, Robinson-O'Brien R et al. (2008) Creating healthy food and eating environments: policy and environmental approaches. Annu Rev Public Health 29, 253-272.

33. Buttenheim AM, Havassy J, Fang M et al. (2012) Increasing supplemental nutrition assistance program/electronic benefits transfer sales at farmers' markets with vendoroperated wireless point-of-sale terminals. I Acad Nutr Diet 112, 636-641.

34. McCormack LA, Laska MN, Larson NI et al. (2010) Review of the nutritional implications of farmers' markets and community gardens: a call for evaluation and research efforts. I Am Diet Assoc 110, 399-408.

35. Glanz K, Sallis JF, Saelens BE et al. (2005) Healthy nutrition environments: concepts and measures. Am J Health Promot 19, 330-333, ii.

36. Gustafson AA, Sharkey J, Samuel-Hodge CD et al. (2011) Perceived and objective measures of the food store environment and the association with weight and diet among low-income women in North Carolina. Public Health Nutr 14, 1032-1038.

37. Franco M, Diez-Roux AV, Nettleton JA et al. (2009) Availability of healthy foods and dietary patterns: the MultiEthnic Study of Atherosclerosis. Am J Clin Nutr 89, 897-904.

38. Hutchinson PL, Nicholas Bodor J, Swalm CM et al. (2012) Neighbourhood food environments and obesity in southeast Louisiana. Health Place 18, 854-860.

39. US Department of Commerce (2010) United States Census Bureau. http://www.census.gov (accessed January 2012).

40. Thornton LE, Pearce JR \& Kavanagh AM (2011) Using Geographic Information Systems (GIS) to assess the role of the built environment in influencing obesity: a glossary. Int J Behav Nutr Phys Act 8, 71.

41. US Department of Agriculture (2012) Building a Healthy America: A Profile of the Supplemental Nutrition Assistance Program. http://www.fns.usda.gov/ora/MENU/Published/ snap/FILES/Other/BuildingHealthyAmerica.pdf (accessed April 2012).

42. Broda C, Leibtag E \& Weinsten D (2009) The role of prices in measuring the poor's living standards. I Econ Perspect 23, 77-97.
43. Glanz K, Sallis JF, Saelens BE et al. (2007) Nutrition Environment Measures Survey in stores (NEMS-S): development and evaluation. Am J Prev Med 32, 282-289.

44. Andreyeva T, Blumenthal DM, Schwartz $\mathrm{MB}$ et al. (2008) Availability and prices of foods across stores and neighborhoods: the case of New Haven, Connecticut. Health Aff (Millwood) 27, 1381-1388.

45. Piernas C \& Popkin BM (2011) Food portion patterns and trends among US children and the relationship to total eating occasion size, 1977-2006. J Nutr 141, 1159-1164.

46. Rose D, Hutchinson PL, Bodor JN et al. (2009) Neighborhood food environments and body mass index: the importance of in-store contents. Am J Prev Med 37, 214-219.

47. Creel JS, Sharkey JR, McIntosh A et al. (2008) Availability of healthier options in traditional and nontraditional rural fastfood outlets. BMC Public Health 8, 395.

48. Babey SDA, Hastert T, Harvey S et al. (2008) Designed for Disease: The Link Between Local Food Environments and Obesity and Diabetes. Los Angeles, CA: California Center for Public Health Advocacy, PolicyLink and UCLA Center for Health Policy Research.

49. Kennedy ET, Ohls J, Carlson S et al. (1995) The Healthy Eating Index: design and applications. J Am Diet Assoc 95, 1103-1108.

50. Morland K, Wing S \& Diez Roux A (2002) The contextual effect of the local food environment on residents' diets: the atherosclerosis risk in communities study. Am J Public Health 92, 1761-1767.

51. Sharkey JR, Johnson CM, Dean WR et al. (2011) Association between proximity to and coverage of traditional fast-food restaurants and non-traditional fast-food outlets and fastfood consumption among rural adults. Int J Health Geogr 10, 37.

52. Bertmann FM, Ohri-Vachaspati P, Buman MP et al. (2012) Implementation of wireless terminals at farmers' markets: impact on SNAP redemption and overall sales. Am J Public Health 102, e53-e55.

53. Kropf ML, Holben DH, Holcomb JP Jr. et al. (2007) Food security status and produce intake and behaviors of Special Supplemental Nutrition Program for Women, Infants, and Children and Farmers' Market Nutrition Program participants. J Am Diet Assoc 107, 1903-1908.

54. Evans AE, Jennings R, Smiley AW et al. (2012) Introduction of farm stands in low-income communities increases fruit and vegetable among community residents. Health Place 18, 1137-1143.

55. Laska MN, Hearst MO, Forsyth A et al. (2010) Neighbourhood food environments: are they associated with adolescent dietary intake, food purchases and weight status? Public Health Nutr 13, 1757-1763.

56. Jilcott SB, Wade S, McGuirt JT et al. (2011) The association between the food environment and weight status among eastern North Carolina youth. Public Health Nutr 14, 1610-1617.

57. Zick CD, Smith KR, Fan JX et al. (2009) Running to the store? The relationship between neighborhood environments and the risk of obesity. Soc Sci Med 69, 1493-1500.

58. Shier V, An R \& Sturm R (2012) Is there a robust relationship between neighbourhood food environment and childhood obesity in the USA? Public Health $\mathbf{1 2 6}$, $723-730$

59. Glanz K, Bader MD \& Iyer S (2012) Retail grocery store marketing strategies and obesity: an integrative review. Am J Prev Med 42, 503-512.

60. Ohri-Vachaspati P, Leviton L, Bors P et al. (2012) Strategies proposed by healthy kids, healthy communities partnerships to prevent childhood obesity. Prev Chronic Dis 9, E11. 